\title{
Germanisch-finnische Lehnbeziehungen: drei Wörter mit fi. -aav- urgerm. -aww- > urn. -aggw-
}

\section{Fi. ha ava 'Wu n d e'}

1.1. Fi. haava 'Wunde' hat Entsprechungen in karel. hoava, olon. huavu, wot. äva 'Wunde' (SKES), estn. haav (Gen. haava) 'Hieb, Wunde' (Wiedemann), liv. ô $v$ 'Wunde' (Kettunen). Aus dem Finnischen ist es ins Lappische entlehnt worden: Vefs.

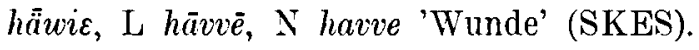

Für dieses ostseefinnische Wort - das im Wepsischen und Lüdischen nicht nachgewiesen ist - hat man früher etỵmologischen Anschluss im Wolgafinnischen gesucht: mordE tšavoms, M šavâms 'schlagen, erschlagen, prügeln', tscherW

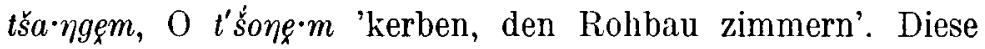
Zusammenstellung ist aber von berufener seite angezweifelt worden. Erkki Itkonen führt in seiner Untersuchung "Zur Geschichte des Vokalismus der ersten silbe im Tscheremissischen und in den permischen Sprachen" (FUF 31, 19531954) diese Verben als solches Wortmaterial an, das keine Entsprechungen im Ostseefinnischen hat, und fügt hinzu: "Dass das fi. Wort haava 'Wunde' hierher gehört, ist nicht wahrscheinlich» (a.a.O. S. 160). Julius Mägiste findet, dass Itkonen diesen Vergleich "wohl mit Fug gestrichen" hat (Commentationes Balticae 8/9 S. 283--284).

1.2. Es liegt nun nahe, auf die Möglichkeit einer alten Entlehnung $\mathrm{zu}$ achten. Und tatsächlich: wenn man sich nach einem passenden Original umsieht, braucht man nicht lange zu suchen: als solches bietet sich germ. *hawwa- > urn. *haggwa-, das in awn. hogg (n.), aschwed., nschwed. hugg usw. 'Hieb' weiterlebt. Dieses Substantiv gehört zum reduplizierenden Verb germ. *hauwan- 'hauen', dessen Fortsetzungen awn. 
hoggva, aschwed., nschwed. hugga, ahd. houwan, (nhd. hauen), ags. hēawan usw. sind und das ursprünglich gerade ein schneidendes, verletzendes Hauen bezeichnet (s. unten und Grimm, DWb: hauen); germ. *hawwa- ist also zunächst 'Hieb'. Wörterbuchangaben zu diesem Substantiv: awn. hogg 'Slag hvormed en rammes; Hug med skarpt Egvaaben, med Øxe, Sverd; Halshugning; Slagtning; Hugst hvormed der fældes Træer; Aabning som fremkommer i et Legeme paa det Sted, hvor det rammes af Hug' (nach Fritzner); aschwed. hug, hugg 'huggning, hygge, rättighet att hugga e. fälla; hugg; hugg, bett; slag' (Söderwall), auch 'hugget sår' (Schlyter).

Die Bedeutung der Fortsetzungen von germ. *hawwa- im Nordischen ist also überall zunächst 'Hieb, Schlag', bes. 'Hieb mit einer schneidenden Waffe'. Durch einen solchen Hieb entsteht aber eine Wunde und so kann mit dem Wort auch eine durch Hieb entstandene Wunde bezeichnet werden: vgl. oben (awn.) 'Aabning som fremkommer i et Legeme paa det Sted, hvor det rammes af Hug', (aschwed.) 'hugget sår', vgl. noch nnorw. hugg, das auch 'innsnitt, hakk, sår frembragt med huggende redskap' bedeutet (Norsk riksmålsordbok). Entsprechend kann das Verb u.a. mit 'durch Hieb verwunden' umschrieben werden: vgl. aschwed. hugga u.a. 'hugga sår, i synn. göra fullsår' (Schlyter): z.B. "Nu dræpær man annan: huggar ælla gör andra akomu." 'Nu dräper någon en annan, sårar honom eller gör honom kroppskada på annat sätt' (Östgötalagen, Vådamålsbalken 9$)^{\mathbf{1}}$; ndän. hugge u.a. 'saare, beskadige ved at hugge' (ODS); oder im Westgermanischen: afries. hāwa 'hauen, verwunden' (Holthausen, Afries. Wb.), dt. schwäb. hauen u.a. 'mit einer schneidenden Waffe draufschlagen, verwunden' (Fischer, Schwäbisches Wb.).

Im Westgermanischen ist das entsprechende Substantiv starkes Maskulinum: mhd. hou 'Holzhieb' (Lexer, Mhd. Handwb.), mnd. houw 'Hau, Hieb' (Schiller - Lübben, Mnd. Wb.). Im Deutschen ist IIau heute schriftsprachlich durch IIieb ver-

\footnotetext{
1 Neuschwedische Übersetzung nach: Svenska landskapslagar, tolkade och förklarade av Åke Holmbäck och Elias Wessén 1 (Uppsala 1933): Östgötalagen S. 77.
} 
drängt worden, mundartlich aber noch erhalten, auch hier verbindet sich 'Hieb' mit 'Wunde': schweiz. Hauw 'Hieb, Hieb- oder Schnittwunde' (Schweiz. Idiotikon 2 Sp. 1801). Vgl. übrigens, dass Wiedemann estn. haav durch 'Hieb, Wunde' und die Wendung haavu saama durch 'Schläge bekommen' übersetzt.

1.3. Das germ. Wort zeigt also, wie 'Hieb' auch 'Wunde' ist. Und so stimmt germ. *hawwa- semantisch mit osfi. haava genau überein, denn es lässt sich aus dem osfi. Material selbst, also sprachintern, erschliessen, dass für haava von der Bed. 'Hieb, Schlag' auszugehen ist, wie eben das finnische etymologische Wörterbuch (SKES) angibt. Osfi. haava wird nämlich -- wenigstens finnisch, estnisch und lappisch - in gewissen Wendungen in der Bedeutung 'Mal, Zeitpunkt' gebraucht, und dieser Gebrauch kann nicht aus 'Wunde', sondern eben aus 'Hieb, Schlag' erklärt werden (vgl. auch solche Parallelen wie lit. kar̃tas 'Mal', eig. 'Schlag, Hieb', slaw. razz 'Mal' $\sim$ raziti 'schlagen' $\sim$ weissruss. vraza 'Wunde' usw.). Beispiele für diesen Gebrauch von haava: fi. tällä haavaa 'diesmal', yhtä haavaa 'mit einem Male, gleichzeitig'; im älteren Finnisch ist der Gebrauch noch allgemeiner, Agricola schreibt z.B.: "Ja cuckoi toisen hauan lauloi» (Markus 14,72: 'Und der Hahn krähte zum zweiten Mal', in der heutigen İ́bersetzung heisst es: "toisen kerran»); estn. haaval 'zur Zeit, auf ein Mal', sel haaval 'dieses Mal', ühel haaval 'mit einem Mal' (SKES, Wiedemann); ähnlich im Lappischen.

Auch diese Bedeutungsentwicklung zu einem Zeitausdruck - und zwar ebenso nur in gewissen Wendungen - teilt germ. *hawwa-mit osfi. haava, und dieser Umstand macht die semantische Übereinstimmung besonders eindrucksvoll: adän. hug auch 'tid, tidspunkt': paa samme hwg (v.J. 1536, Kalkar, Ordbog til det ældre Danske Sprog); schwed. hugg auch 'tillfälle, stund': det skedde just $i$ det hugget (SAOB), schwed., gotl. $i$ samma hugget 'i det ögonblicket, just då' (Säve et al., Gotländsk ordbok). Vgl.: genall so afi. samalla haavalla: "-- nijn hän cohta samalla haawalla saij hänen nägöns" 'so bekam er gleich in demselben Augenblick sein Augenlicht wieder' (Bischof Sorolainen, Postilla v.J. 1621, S. 484). - Es ist auch 
möglich, dass dieser Gebrauch als fertiges Muster aus dem Germanischen ins Ostseefinnische übernommen wurde; wenn so, dann wäre nicht unbedingt nötig anzunehmen, dass osfi. haava selbst 'Hieb' bedeutet hat, es würde auch genügen, dass sein germ. Original diese Bedeutung hatte.

1.4. In (ur)germ. *hawua- haben wir also die bekannte urgermanische Verschärfung - $w w$-, die sich später im Nordgermanischen und Gotischen zu -ggu- weiterentwickelt hat. Geht nun osfi. haava auf urgerm. *havica- oder auf die etwas spätere urnordische Form *haggica- zurück? ${ }^{1}$ Die Entscheidung ist wohl nicht mit Sicherheit zu treffen: lautlich ist beides möglich, wie anschliessend näher ausgeführt wird. Einen Parallelfall für die finnische Behandlung von germ. $-w w->$ urn. -ggwhaben wir in dem bekannten Verhältnis fi. kuva 'Bild' germ. *skuwwan- > urn. *skuggwan- > awn. skuggi 'Schatten, Spiegelbild, Spuk'. - Ich betrachte zuerst den Ansatz *hawwa-.

Es gab offenbar im (Früh)urfinnischen keine Gemination *-vv-, so dass die germanische Verschärfungsgemination -wwnur mit éinem $-v$ - wiedergegeben wurde: vgl. kuva. Man bedenke, dass z.B. fi. sauva 'Stab' nicht auf älteres *savva, sondern auf *śavna zurückgeführt wird (SKES). Zu einer Zeit, als noch *savna (bzw. *sauna o. ähnl.) für heutiges sauva gesprochen wurde, gab es also offenbar kein *-auv- (bzw. *-avv-) in der Sprache, jedenfalls kann man das Gegenteil nicht beweisen. ${ }^{2}$ Ein urfi. *hauva (= heute ebenfalls *hauva) wäre also

1 Es sei vermerkt, dass "Urgermanisch" und "Urnordisch" als chronologische Begriffe zu verstehen sind. Auch im Norden - und gerade im Norden - wurde einmal Urgermanisch gesprochen, das sich dann allmählich zu einer Urnordisch genannten Sprachform entwickelte. Die lautlichen Unterschiede zwischen den beiden Sprachstufen sind geringfügig, so dass die Stufen in etym. Darstellungen oft nicht auseinandergehalten werden. Urnordisch ist die gemeinsame Vorstufe der späteren nordischen Einzelsprachen (Alt(west)nordisch = Altisländisch, Altnorwegisch; Altostnordisch $=$ Altschwedisch, Altdänisch).

2 Wörter wie fi. neuvo 'Rat; Gerät' (= estn. nõu = liv. ne'u) und fi. auvo (veraltet) 'Freude; Ehre' (= estn. $a u=$ liv. o' $u(o)$ ) können kein ursprüngliches (frühurfi.) *-ov- beweisen, weil ihre Verbreitung sich auf das Osfi. beschränkt: lp. nxo'vo ist (nach SKES) aus dem Finnischen entlehnt und auch für lp. avvo erwägt Y. H. Toivonen (SKES) 
für germ. *hawwa- überhaupt erst seit der Zeit möglich gewesen, als es schon die fi. Sequenz -auv- usw. gab (sauva, neuvo 'Rat'). Aber selbst dann wäre die Wiedergabe von *hawwa- durch haava wohl adäquater oder wenigstens ebenso adäquat gewesen, denn die Entwicklung -aww- >-aggw- setzt ja voraus, dass - $u w$ - recht konsonantisch gesprochen wurde.

Ein Quantitätsunterschied im Vokalismus - hier germ. Kürze osfi. Länge - ist bei zahlreichen Lehnwörtern anzutreffen. Wir haben sowohl germ. Länge $\sim$ fi. Kürze als auch, wie hier, das umgekehrte Verhältnis: für dieses vgl. germ. *wikōn- fi. viikko 'Woche', germ. *alðōn- fi. a(a)lto 'Welle', germ. *justa- fi. juusto 'Käse'. Die Länge kann hier ausserdem dadurch erklärt werden, dass man die germ. Silbenlänge (lange Silbe *haw-) hat beibehalten wollen. - $\mathrm{Zu}$ erinnern wäre schliesslich auch daran, dass die Quantitätsopposition bei $a$ im Vorfinnischen nicht nachzuweisen ist. Wenn die Entlehnung so früh geschehen wäre, dass sich noch keine deutlich empfundene Opposition $/ \mathrm{a} / \neq / \overline{\mathbf{a}} /$ herausgebildet hätte, könnte sich die Länge (bzw. die Kürze) erst mit der zunehmenden Schärfe dieser Opposition festgesetzt haben (vgl. fi. kaava estn. kava unten: 2.1.). Aber diese Erklärung ist in unserem Fall natürlich entbehrlich. Die vielfach auftretenden Quantitätsunterschiede bei den Entlehnungen im Vergleich zu ihren Originalen werfen aber immerhin ganz allgemein die Frage auf, in wiefern sie etwa durch undeutliche bzw. ganz fehlende Oppositionen in frühen Stadien des Finnischen zu erklären sind. ${ }^{1}$

Wenn wir dagegen von der urn. Lautgestalt *haggwa- ausgehen, ist urfi. $-\bar{a} v$ - hier einfach als Lautsubstitution für urn. -aggu- anzusprechen (es ist anzunehmen, dass -gg- zuerst noch

dieselbe Möglichkeit. Lauri Kettunen erklärt übrigens auvo aus älterem *avo (wofür bes. das Livische sprechen soll) und auch für neuvo hält er dieselbe Entwicklung für möglich (Vir. 1956 S. 137-, Mémoires de la Soc. Finno-ougrienne 119 S. 140, 141). Vgl. auch dial. sauvu, savvu, sauhu für norm. savu 'Rauch' (SKES). - Wörter wie rouva 'Frau', touvi 'Tau' (jüngere Lehnwörter), vauva 'Kleinkind' oder gar hauøa 'Hund' (Kinderspr.) kommen natürlich überhaupt nicht in Betracht.

1 Tuula Niskanen und Tette Hofstra machen auf den häufigen Quantitätswechsel aufmerksam in UAJb. $43 \mathrm{~S} .51$. 
spirantisch war: $-\gamma \gamma$-). Denn ein zunächst entsprechendes urfi. *-aki- wäre ja unmöglich gewesen, weil im Urfinnischen die Sequenz *-kv- sicher nicht vorkam, wie sie auch heute nicht möglich ist. - Vgl. übrigens hierzu den bekannten diachronen Vorgang z.B. *takja > taaja 'dicht' (in der finnischen Sonderentwicklung).

Da wir hier für germ. $h^{-}\left(\chi^{-}\right)$fi. $h^{-}$haben, ist haava (nach gängiger Meinung) als eine etwas jüngere Entlehnung anzusehen als diejenigen Wörter, die für germ. $h-(\chi-)$ fi. $k$ aufweisen (z.B. fi. kana 'Huhn'). Dieses $h$ - (wie bei hartia, 'Schulter', hame 'Rock', hipiä 'Haut(farbe)' u.a. Entlehnungen) könnte für eine urnordische Entlehnung sprechen. Wir können aber nichts Sicheres behaupten, schon weil die Zeit für

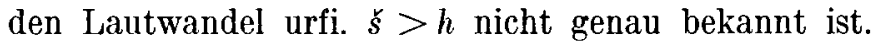

Für das hier aufgespürte germ.-fi. Lautverhältnis lassen sich noch Parallelfälle finden: sie werden unten behandelt.

2. Fi. kaava' $U \mathrm{mr}$ is s, Gestalt, Form, II u ter'

2.1. Fi. kaava ist in der heutigen Gemeinsprache zunächst als 'Muster, Formular' bekannt, es hat aber seltener auch die allgemeinere Bedeutung 'Gestalt, Form, Umriss' (NS ['Wb. der fi. Gegenwartsspr.']: 'kuvio, hahmo, (luonnos)piirros': »- - avatusta ovesta virtas sisään auringon heleä paiste, kuvaten kultaisen k:an tuvan lehditetylle laattialle» (A. Kivi)). In SKES wird es mit 'malli, ulkopiirteet' umschrieben. Ältere Wörterbuchangaben: 'mall, mönster / forma' (Ganander), 'formula 1. exemplum cujus ad formam quid conficitur / Muster, Form' (Renvall), 'form, formel, formulär; modell, mall, mönster (malli); schema, föreskrift' (Lönnrot). Ein alter Beleg zeigt kaava als 'Schattenbild, Körperhülle, Leiche': scawas tuossa Nytt on, mutta cusa sielus liene, dhett ähr, att Skuggan af din Kropp ähr fulldähr, men hwar Siählen må wara» (Liminka, aus dem Gerichtsbuch von Nordostbottnien v.J. 1680, Wörterbuchstiftung). Auch heute wird ein bleicher, krank aussehender Mensch dial. kuoleman kaava (auch $k: n$ kaave) genannt (Laihia, Oulujoki u.a.). In den heutigen Mundarten ist kaava zunächst - 
wie in der Gemeinsprache - 'Musterstück, Modell' (wonach etwas verfertigt wird).

Es kommt auch die Form fi. kaave vor: 'Gesicht, Gespenst': "elä mää sinne saunaa makoomaa, määttiijä mitä kuaveita siellä näyttäätyỳ" ('geh nicht in die Sauna schlafen, da können sich womöglich Gespenster zeigen', Kitee; sonst wenig Belege in den heutigen Maa.), kuoleman kaave (vgl. oben) sagt man von einem blassen, krank aussehenden Menschen (also 'einer, der wie ein Toter aussieht', bes. aus SW-Finnland bezeugt); kaave bedeutet weiter 'Balban = Vogelbild, Lockvogel' (SW-Finnland, nordwestlich von Turku); zunächst im Pl. (kaaveet) ist das Wort sporadisch für 'Kleider' belegt, vorwiegend östlich ist die (pluralische) Verwendung für 'Manieren; Possen, Streiche' und ganz allgemein für 'Sache(n), Gerät(e), Werkzeug(e)' u.dgl. ${ }^{1}$

${ }^{1}$ In denselben östlichen Mundartgebieten wie kaave 'Sache(n)' u.dgl. ist auch ein kurzvokalisches kave (Pl. kaveet) 'Arbeitsgerät, Ding, Siebensachen, bewegliches Eigentum' u.dgl. belegt (Wörterbuchstiftung). - Es gibt aber auch ein fi. kave mit Stufenwechsel (Gen. kape(h)en) 'Geschöpf, Mensch, Mädchen', kapo ds. (Volksdicht.); kave ist dial. auch 'Schaf' (mundartl. kaves, in (Süd)ostbottnien), hierzu auch südestn. kabo, kabe, kabene u.dgl. 'Frau, Weibsperson, Mädchen' und lp. (N) gābâ (< urfi. *kaßek?, s. näher SKES unter kave). Für dieses letztere kave(h) 'Geschöpf, Schaf' usw. ist schon früher ie. Herkunft vermutet worden: ie. *skab- 'schaffen' (SKES; V. Ruoppila findet diese auf E. N. Setälä zurückgehende Etymologie plausibel: Kotieläinten nimitykset suomen murteissa I S. 199, SKST 222). Wir können diesen offenbar richtigen Ansatz noch prāzisieren: kave( $h)$ dürfte zurückgehen auf germ. *skapi-z, das lautlich genau als Original passt; germ. *skapi-z lebt weiter in ahd. skaf 'Beschaffenheit', als Nachsilbe ('Beschaffenheit') in awn. -skapr, ags. -sciepe, as. skepi usw.; dieses verbreitete germ. Wort gehört eben zu germ. *skap- 'schaffen', d.h. zu der genannten ie. Sippe (s. z.B. E. Seebold, Verzeichnis u. etym. Wb. der germ. st. Verben, The Hague - Paris 1970, S. 407). Zu derselben Sippe gehört etwa auch ags. ge-sceap 'Geschöpf', dt. Ge-schöpf usw. Semantisch vgl. noch etwa estn. loodus 'Geschöpf, Geschaffenes', aber auch 'Gestalt, Beschaffenheit' (Wiedemann). Das Lautverhältnis urgerm. - p- < vorgerm. $-b$ - urfi. $-p$ - (statt $-p p-$ ) spricht für eine sehr frühe Entlehnung. Das lp. Wort weist auf urfi. *-ek (u. nicht auf $*_{-}^{*} s$ ). Es kann hier ein Übergang derselben $A r t$ vorliegen wie bei den germ. Entlehnungen fi. vierre (<*-ek) 'Bierwürze' (< germ. *wertez), vaate 'Kleid', ruoste 'Rost' (s. auch Hakulinen, SKRK, 3. Aufl., S. 103). In diesem Zusammenhang 
- Neben kaave ist früher auch kaavis vorgekommen, schon Juslenius hat caauis, -en 'funus; spectrum / lijk; spöke'. Andere Wörterbuchangaben: Ganander hat für kaawis, -wen dieselbe Erklärung wie Juslenius, erläutert aber noch das Wort mit kejjuset (die keijuset waren eine Art Totengeister); Renvall: kaawet, een al. kaawi, wen 'corpus hominis mortuum / Leiche; inde umbra hominis 1. spectrum, manes / Todtenerscheinung, Gespenst'; Lönnrot: kaawe, kaaween (kaawi) 'lik; bulvan; spöke, vålnad', kaawis, kaaween = kaawi (?), (Nachträge:) kaawe, kaaween 'vette, sjöfågelvette, lockfågel (uikukko)', Pl. kaaweet 'intriger; rustning, beklädnad, klädsel (tamine)'. - Als verbale Ableitung gehört hierher u.a. dial. kaavehtia 'sich undeutlich zeigen, spuken' (Ostmaa., Wörterbuchstiftung).

Fi. laava mit seiner Sippe hat Entsprechungen im KarelischOlonetzischen, Lüdischen, Wotischen (?) und Estnischen. Karel.olon. hoava, kuavu bedeutet erstens 'malli, esikuva; laatu, laji' ('Muster, Vorbild; Art'), beachtenswert ist aber, dass auch die Bed. 'Zeichen' ('merkki, viitta') belegt ist: dorogan kuavu 'Wegzeichen', nuotan kuavu 'Netzzeichen' (Säämäjärvi, KKS ['Wörterbuch des Karelischen']). Es kommen ferner mehrere Verbalableitungen hinzu: z.B. koavehtie 'kuvitella; kuvastella, kangastella mielessä; kummitella' ('sich (etwas) vorstellen; sich (undeutlich) zeigen; spuken' u. dgl., KKS), koacastuo 'ilmestyä, tulla näkösälle' ('erscheinen, sich zeigen', KKS).

Im Estnischen hat die Entsprechung von fi. laava kurzes -a-: liava 'Schema, Formular, Disposition' (Wiedemann). Dem fi. kaave entspricht wot. (Volksdicht.) kaave 'hahmo, näkö, kauneus' ('Gestalt, Anblick, Schönheit', aus dem Fi.?: SKES)

ist es ferner nicht uninteressant, auf das westgerm. Wort für 'Schaf', westgerm. *skäpa- < urgerm. *skēpa- hinzuweisen (= dt. Schaf, engl. sheep usw.). Das Wort gilt als nicht etymologisiert. Nach den ie. Ablautregeln könnte es aber eine Ableitung von germ. *skap-darstellen, also als 'Geschöpf' gedeutet werden: vgl. eben fi. dial. kave 'Geschöpf, Schaf' u. schwed. kreatur 'Tier, Geschöpf', dial. auch 'Schaf' (das letzte Beispiel nach Ruoppila a.a.O). - Es wäre wohl möglich, dass die fi. - $p$ - Gruppe sich sekundär mit der stufenwechsellosen - - -Gruppe (ka(a)ve) z.T. vermischt hat: vgl. etwa fi. kapine, das semantisch zu kave 'Sache' passt. Dann könnte fi. östl. ka(a)ve 'Sache' ursprünglich zu der -pGruppe gehört haben. 
und estn. veralt. kaave 'Gespenst' (Wiedemann). Eine verbale Ableitung ist lüd. kuavehtida 'häämöttää' ('sich undeutlich zeigen' $=$ fi. kaavehtia, SKES). Aus dem Fi. oder Karel. ist lpKo. kāvas, Kld. kavas, T kāu's 'Heiligenbild' (= fi. kaave $(h)$ ) entlehnt (SKES).

2.2. Die verschiedenen Gebrauchsweisen von kaava - kaave lassen sich zunächst auf einen gemeinsamen Inhalt, ungefähr 'äussere Form, Hülle, Aussehen, Gestalt, Anblick, den etwas gewährt' vereinigen. Dazu gesellt sich dann die karel.-olon. Bedeutung 'Zeichen'. Wie sich 'Zeichen' auch etwa mit fi. dial. (SW) kaave 'Lockvogel' verbindet, zeigt interessanterweise schwed. vette 'Lockvogel; (See)zeichen'. -- Schon E. N. Setälä meinte, die ursprüngliche Bedeutung von kaava sei 'Aussehen, Gestalt' gewesen ('ulkopiirteet'), kaave habe ursprünglich 'Gesicht' ('näkỵ') gemeint (Sanastaja 1927 Nr. 2 S. 10-). Semantisch stimmt das alles zu den germ. Substantiven um germ. *skaww - (verbal in ahd. scouwōn, ags. scēawian 'schauen', nengl. show 'zeigen'): ahd. scou (Geschlecht unbek.) 'habitus', also 'Gestalt' (Graff, Ahd. Sprachschatz 6 Sp. 556, Trübners Dt. Wb. 6 S. 36), mhd. schou (m.), Gen. schouxes 'der Anblick, den etwas gewährt', schouve (f.) 'suchendes, prüfendes Schauen, Blick; das, was gesehen wird, Anblick, den etwas gewährt, Aussehen, Gestalt': vgl. etwa: "se in swacher schouwe, nu ein rīchiu frouwe» zuvor [hatten Sie] ein dürftiges Aussehen (wörtlich: [waren Sie] "in dürftiger Gestalt»), jetzt [sind Sie] eine mächtige Frau' (Hartmann von Aue, Erec 6475, Lexer, Mhd. Handwb. 2 Sp. 775, 777). Im Mittelniederdeutschen ist die der karel.-olon. Sonderbedeutung entsprechende Bedeutung 'Zeichen' belegt: mnd. schow (n.) 'sichtbares Notzeichen' (Schiller - Lübben, Mnd. Wb. 4 S. 130).

Es sind also Substantive aller drei Genera überliefert worden, es ist wahrscheinlich, dass schon im Urgermanischen mehrere Formen existierten. Den schriftlich überlieferten germ. Formen entsprechen in urgerm. Rekonstruktion folgende Stämme: dem Femininum der $\bar{o}$-Stamm *skawwō- < älter *skaww $\bar{a}-$ (auch *skauuiō- wäre theoretisch möglich), dem Maskulinum entweder der $a$-Stamm *skauwa- (der auch dem Neutrum entspricht) oder auch der $i$-Stamm *shauwi-: die mhd. (und ahd.) 
Lautgestalt lässt beide Möglichkeiten zu. Auch die finnischen Formen lassen sich aus verschiedenen germ. Originalen erklären: wie mehrere andere germ. Lehnwörter auf -a (fi. laita, neula usw.) könnte fi. kaava auf das Femininum zurückgehen, aber natürlich ist auch der maskuline (und neutrale) $a$-Stamm als Original möglich.

Osfi. kaave - kaavis sind bisher als Ableitungen von kaava erklärt worden. Diese Erklärung wird durch die germ. Etymologie nicht entkräftet. Anderseits muss aber festgestellt werden, dass kaave und kaavis diejenigen beiden Stammtypen repräsentieren, die gewöhnlich zur Wiedergabe der germ. $i$-Stämme gedient haben: vgl. fi. palje (Gen. palke(h)en) '(Blase)balg' $\sim$ germ. (Nom. Sg.) *balyi-z, fi. kaunis 'schön' $\sim$ germ. *sliauni-z. ${ }^{1}$ Es ist somit auch mit der Möglichkeit zu rechnen, dass kaave und kaavis unmittelbare Entlehnungen aus dem Germanischen sind. Als ihr Original wäre dann germ. (Nom. Sg. $i$-St.) *skawui-z anzusetzen. $i$-Stämme sind - neben $a$-Stämmen - häufig bei germanischen Verbalsubstantiven. Zum Nebeneinander kaave - kaavis vgl. das ebenfalls aus dem Germ. stammende Paar fi. tarve - tarvis 'Bedürfnis'.

Schon Setälä äusserte die Vermutung, fi. laava könnte mit jener germ. Sippe, zu der dt. schauen gehört, zu verbinden sein (Sanastaja 1927, ebenda). Er brachte jedoch noch keine genauere Zusammenstellung.

2.3. Zum lautlichen Verhältnis zwischen den germ. und fi. Formen gilt im übrigen das zu haava Gesagte (1.4.). Auch zur alternativen Herleitung aus dem Urnordischen kann das dort Gesagte unverändert bleiben, denn der Umstand, dass germ. *skawwa- usw. in dieser Ablautform in den späteren nordischen Einzelsprachen nicht mehr vertreten ist, hindert uns nicht, ein frühes urn. ${ }^{*}$ skaggw- anzusetzen (s. unten 2.4. u. 2.5.).

2.4. Urgerm. *shauwa- u.dgl. ist also in literarischer Zeit nur im sog. Westgermanischen vertreten. Wir kennen mehrere andere germ. Lehnwörter im Finnischen, deren Originale nur noch im Westgermanischen überliefert sind (s. meinen Artikel

1 Aber auch bei kaavis scheint die weitere Flexion nach kaave gegangen zu sein: Gen. kaaveen (siehe die Wörterbuchangaben in 2.1.). 
in Neuphil. Mitteilungen 1973 S. 608). Solche Wörter sind wohl kaum aus einer frühen westgerm. Mundartform entlehnt, vielmehr zeigen sie zunächst nur, dass die Entlehnungen aus einer Zeit stammen, als sowohl die Verbreitung der Wörter wie auch die Wohnsitze der Vorfahren späterer germ. Stämme noch anders verteilt waren als später: diese "westgerm." Wörter haben einmal auch an der Ostsee gelebt.

2.5. Die etymologische Sippe, zu der germ. *skaww- gehört (ie. * $(s) k e u$ - 'schauen'), ist umfangreich, und sie hat auch zahlreiche Vertreter in den nordischen Sprachen: u.a. awn. skygn 'sehend', skygna 'schauen', skoða 'schauen, untersuchen', auch germ. *skau-ni-z 'schön' gehört hierher: vgl. bes. got. guda-skaunei 'Gottesgestalt' (Jan de Vries, An. etym. Wb., IEW). Umstritten ist die Zugehörigkeit von germ. *skuwwan- > awn. skug$g i$ 'Schatten, Spiegelbild, Spuk' gewesen. Als alternative Möglichkeit galt die Herleitung von ie. *skeu- 'bedecken'. Das hier besprochene finnische Material spricht nun dafür, dass die erste Alternative das Richtige trifft. Fi. kuva 'Bild' geht ja bekanntlich auf dies germ. *skuwwan- zurück, und kuva steht kaava, kaave semantisch sehr nahe: vgl. fi. dial. kuva auch 'Lockvogel' (wie kaave), lüd., weps. kuva auch 'Schuhleisten' (SKES) fi. kengän kaava 'Schuhleisten' (Renvall, Lönnrot). Germ. *skawwa- und *skuwwa- gehören also offenbar zusammen (so neuerdings auch Fr. O. Lindeman). *skuww- kann übrigens als eine erst analoge Bildung $\mathrm{zu} *$ skaww- angesehen werden, denn ursprünglich wäre in der hier vorliegenden Schwundstufe nur -uw- (nicht -uuw-) möglich. ${ }^{7}$ In der Sprachform, die *skuwwgekannt hat, muss demnach einmal auch *skaww- vorhanden gewesen sein.

3. Fi. $n a a v a$ 'Bartflechte'

3.1. Fi. naava 'Bartflechte (am Baumstamm)' hat gleichbedeutende Entsprechungen in karel. noava, lüd. ńuav (SKES).

1 Diese Erklärung bietet Fredrik Otto Lindeman, Les Origines indoeuropéennes de la "Verschärfung» germanique (Oslo 1964) S. 162, S. $169-170$. 
Aus dem Lappischen gehört hierher (SKES) lpL nävva 'fjun, fint hår / Flaum, feines Haar, fint, mjukt, nyväxt hår' (Grundström), R nav, nava 'höyty, nöyhtä; naava' ('Flaum, Flocke; Bartflechte': SKES; wo ist 'Bartflechte' belegt?), Arj. nāvva 'Haar am menschlichen Körper (nicht am Kopf)' (Lagercrantz). Nach SKES kann dieses lp. Wort aus dem Finnischen entlehnt sein. Zum Verhältnis 'Haar' 'Bartflechte' kann schon das deutsche Wort Bartflechte vergleichsweise herangezogen werden, weiter auch fi. haven 'Haar', dial. auch 'Bartflechte' (SKES, Wörterbuchstiftung). - U̇ber ein paar andere lp. Wörter, die nach SKES möglicherweise auch hierher gehören, siehe 3.4.

Diesen Wörtern steht nun schwed. (dial.) fnugg (n.) - dial. Nebenform fnagg - semantisch nahe: fnugg ist 'fjun, ludd, flock', (früher auch '(kringflygande) stoft o. damm'), första fniugget på hakan ('erster Flaumbart am Kinn', v.J. 1745, -iist wohl durch fiun beeinflusst), man sagt es auch von 'kort gräs som är svârt att bärga' (SAOB). Dial. fnagg ist 'kort gräs; kort hår' (Rietz). Sowohl schwed. fnugg als auch lp. nāvva haben also die Bed. 'fjun', 'feines, weiches Haar' gemeinsam, und auch sonst ist die semantische Übereinstimmung gross. Die gemeinsame Bed. '(feines) Haar' ist offenbar auch älter als die spez. finnische Bed. 'Bartflechte', weil eben 'Haar' > 'Bartflechte' natürlicher ist als die umgekehrte Richtung. Mit schwed. fnugg offenbar identisch ist dän. fnug (n.) 'feines Haar' (nach ODS genauer: 'lille, let og fin bestanddel af noget, om de smaa haar ell. dun, hvormed frugten hos visse planter føres omkring af vinden, (nu l. br.) om den første, bløde skægvækst; dun; laadden, fnugget ell. dunet overflade af ell. overtrack paa noget').

3.2. Schwed. fnugg kann nun auf germ. *fnawwa- zurückgehen, wie schwed. hugg auf germ. *hawwa- zurückgeht. Diese Etymologie wird in der Forschung auch allgemein angeführt (Tamm, Falk - Torp, Hellquist, neuerdings Fr. O. Lindeman, s. unten). Wir können also fi. naava von diesem germ. Wort herleiten. Weil unser Wort aber nicht im Westgermanischen vertreten ist und weil auch kein awn. Beleg vorhanden ist (awn. müsste es * fnogg lauten), haben wir für den Ansatz germ. -ww- keine absolute sprachinterne Evidenz. In dieser Beziehung unterscheidet sich also unsere dritte Zusammenstellung von 
der ersten und zweiten, in denen germ. -ww- eindeutig feststeht. Wir haben jedoch ein Indiz für germ. -ww- in der dial. Nebenform fnagg. Diese Form kann sich nämlich so zu fnugg verhalten wie schwed. dagg 'Tau (m.)' zu schwed. dugg 'Tauregen', (dial. 'Tau': beides < germ. *dawwō-), oder schwed. dial. hagg, hagga zu hugg, hugga. Beide Formen können also auf germ. * fnawwa- zurückgehen; neben dem mundartlich erhaltenen alten - $a$ - in fnagg stellt das - $u$ - in fnugg dann eine normale Entwicklung dar, welche Entwicklung eben in Wörtern mit altem - $w w$ - vor sich gegangen ist. Es wäre auch möglich aber wohl kaum wahrscheinlich - , dass nur fnagg ein altes ${ }^{*}$ fnawwa- vertritt, fnugg aber ${ }^{*}$ fnuwwa- (s. Hellquist). - Wenn wir dagegen etwa ein altes $-g g^{-}$(d.h. ein schon urgerm. $-\gamma \gamma-$ ) annehmen würden, könnte das Nebeneinander fnugg-fnagg nicht als alter Ablaut erklärt werden: es gibt keinen genuinen Ablaut germ. $a: u .^{1}$ Auch Fr. O. Lindeman setzt germ. ${ }^{*}$ fnaw$w a-$ an. ${ }^{2}$

3.3. Germ. *fnawwa- ist also schon im Lichte des sprachinternen Materials nicht nur möglich, sondern auch wahrscheinlich. Und zu diesem Ansatz passt nun auch unser fi. naava semantisch und lautlich. Wenn wir hier ein typisches Kulturwort vor uns hätten, wäre die Entlehnung aus dem Germanischen hiermit sicher und der germ. Ansatz wäre dadurch bestätigt. Im vorliegenden Fall kann man zunächst von Wahrscheinlichkeit sprechen. Aber die Wahrscheinlichkeit wird eben dadurch erhöht, dass wir dasselbe Lautverhältnis schon bei haava und kaava haben, wo der Ansatz germ. -aww-feststeht. - Unter den mit fi. naava bedeutungsähnlichen Wörtern finden sich übrigens mehrere Lehnwörter, angefangen mit fi. larva 'Haar' (balt. Lw.), weiter hahta, hahtu, hahdun (Gen. hahtumen) 'feines Haar, Flaum, Flocke', hahtuva 'gekämmte Faserflocke (Wolle, Flachs)' < germ. *hazda- (> awn. haddr

${ }^{1}$ In Fällen wie germ. *skawwa: *skuw( $(w)$ a handelt es sich dagegen um regelrechten Ablaut: die erste Form stellt ie. o-Hochstufe, die zweite ie. Schwundstufe dar.

2 Fredrik Otto Lindeman, Les origines indo-européennes de la "Verschärfung" germanique (Oslo 1964) S. 167. Freilich hat er hier nichts Neues zu bieten. 
'Kopfhaar der Frau', in SKES "vielleicht»); dass auch im Germanischen von 'Faser' und Faserverarbeitung auszugehen ist, zeigt das ablautende germ. *hezdō(n)->ags. heordan (Pl.) 'Werg' (engl. hards ds.), mnd. herde 'Flachsfaser', mnd. hëde, nhd. Hede 'Werg' (auf Faserverarbeitung weisen auch die aus-

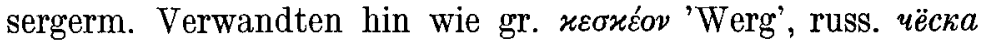
ds.). Ein neueres Lehnwort ist z.B. fi. nukka 'Flor; feines Haar, Flaum' änschwed. nocka, nåcka, nock 'Flor, Noppe, Flocke' (SKES).

3.4. Es sind noch ein paar lp. Wörter als $\mathrm{mögliche}$ Entsprechungen für fi. naava vorgeschlagen worden (SKES): L njā vve 'die langen Haarzotten, die Mähne unter dem Hals des männlichen Rentieres' (Grundström); das Wort kommt mit derselben Bedeutung auch im lpN (njavve) und lpI vor; dann eine zweite vorgeschlagene Möglichkeit: $\operatorname{lpN}$ nuov've (auch nuovve, beides ohne Stufenwechsel) 'tow which one makes by picking to pieces, fraying, rope; tow for cleaning (e.g.) guns' (Nielsen), also 'Werg', IpL nuovvē 'Hanfabfall, der verfitzte, verhedderte Hanf, der bei der Verarbeitung übrigbleibt' (Grundström), lpKo. nuaves hat dieselbe Bed. wie das $\operatorname{lpN}$ Wort (T. I. Itkonen); als drittes Wort ist lpKo. ńŭəuve 'Brachsenkraut, Isoëtes' (SKES) angeführt, das aber auch mit $n$ belegt ist ( $\mathrm{T}$. I. Itkonen, Wb. des Kolta- und Kolalappischen S. 311 a, S 290 b). Die Zusammenstellung mit nuov've stammt von K. B. Wiklund (Urlp. Lautlehre S. 130), die zwei anderen von T. I. Itkonen (JSFOu. 32,3, S. 27).

Was den Vokalismus angeht, so verträgt sich fi. naava mit der Möglichkeit einer Zusammenstellung mit lp. nuov've 'Werg' (und auch mit dem ostlp. Wort für 'Brachsenkraut') - allerdings unter der Bedingung, dass in der ersten Silbe ein ursprüngliches kurzes - $a$ - angenommen wird (vgl. E. Itkonen, Vir. 1950 S. 74); Lönnrot kennt übrigens auch fi. dial. nava 'naava', wo ist es aber sonst belegt? Das Lautverhältnis fi. $a \sim$ lp. $u_{0}$ (in der ersten Silbe) ist nämlich auch sonst bei alten germ. Entlehnungen anzutreffen: fi. rauta 'Eisen' $\sim$ lp. ruow'de ds. < germ. 'rauðan->awn. rauði 'Rasenerz', fi. vartoa 'warten' $\sim$ lp. vuor'det ds. $<$ germ. *warðö- (Nebenform ${ }^{*}$ warde-) $>$ dt. warten usw., fi. tarpoa 'Fische (mit einer 
Stange) aufstören' $\sim$ Ip. duor'bot ds. < germ. *staur- in ${ }^{*}$ stauria- $>$ dt. stören und in *staura- $>$ awn. staurr 'Stange'l und andere mehr. Eine andere Sache ist es dann, dass Konrad Nielsen diese fi.-lp. Zusammenstellung wegen der Unveränderlichkeit des lappischen Stammes beanstandet und für das lp. Wort die Herleitung von einem urn. Substantiv *nōwia- (n.) vorschlägt (K. Nielsen, Studia Septentrionalia 1, Oslo 1945, S. 61). Für dieses angenommene urnordische Substantiv *nōwiagibt Nielsen aber gar keine Fortsetzung in überlieferten Sprachstufen an (nur das entsprechende Verb), so dass sein Ansatz ad hoc konstruiert ist (er $\mathrm{k}$ ö $\mathrm{n} \mathrm{n} \mathrm{t} \mathrm{e}$ natürlich trotzdem das Richtige treffen, wenn wirklich das Fehlen des Stufenwechsels eine Entlehnung aus dem Urn. nahelegen sollte: semantisch wäre die Übereinstimmung mit dem Verb gut: vgl. awn. gnua 'reiben'). T. I. Itkonen hat übrigens hierzu bemerkt, dass es im Ostlappischen die Form mit $v v \sim v$ gibt (das oben erwähnte nuaves: s. näher JSFOu. 60,5 S. 13-14), ohne aber den ad hoc -Charakter des Nielsenschen Ansatzes zu merken.

Was nun lp. njavve betrifft, so ist das mouillierte $\dot{n}$ - vom Standpunkt unserer germ. Zusammenstellung nicht die zunächst zu erwartende Entsprechung für fi. n-. Es müsste nach normalem Lautverhältnis dieselbe Mouillierung im Frühurfinnischen voraussetzen. Bei einem germ. Lehnwort wäre aber eine solche frühe Mouillierung befremdend, und auch die Entlehnungszeit müsste dann sehr früh angesetzt werden. Aber die lp. Mouillierung könnte vielleicht auch als hypokoristischexpressiv aufgefasst werden und brauchte so nicht uralt $\mathrm{zu}$ sein. Die karelische und lüdische Mouillierung bei naava ist eben von dieser Art; diese Mouillierung kommt auch bei Lehnwörtern vor: z.B. karel., lüd. niaba 'Nabel' (balt. oder germ.

${ }^{1}$ Diese Zusammenstellung ist neu und stammt von mir (ausführlicher soll sie in einem anderen Zusammenhang behandelt werden); zur Metathese vgl. fi. karva 'Haar' balt. *gaura- und fi. sarpa, sarva 'Binse' germ. *saura- (neue Zusammenstellung; vgl. SKES unter fi. saura). Die Sequenz *-aur- (*avr-) war offenbar im Frühurfi. unmöglich. - Nach Fertigstellung dieses Manuskripts habe ich noch weitere Zusammenstellungen dieser Art (d.h. fi. $a \sim$ lp. uo bei germ. Entlehnungen) vorgenommen, die ich an anderer Stelle behandeln werde. 
Lw., s. E. Itkonen, Kieli ja sen tutkimus, Helsinki 1966, S. 203 -206). Mit etwas Ähnlichem dürfte also auch für das Lappische gerechnet werden können. ${ }^{1}$ - Nebenbei: wie ist $\operatorname{IpN}$ $n \tilde{a} v v \hat{a} \sim$ nâvvâ 'rubbish, refuse; as the first part of compounds: very short, stunted, and poor (grass, wool etc.)' (Nielsen) zu beurteilen? Friis hat navva, nava $\mathrm{s}$. = naltta, das letztere Wort ist 'truncus, stipula / en kortvoksen Gjenstand, Stump, Stub, som staar igjen efter afhugget Tre, afklippet Haar etc.' (auch hier ein Lehnwort mit -uw-im germ. Original?: vgl. awn. snoggr 'kurz, kurzhaarig', schwed. dial. snugg 'kort', norw. snøgg 'kort, knapp' usw. < germ. *snawwa-, *snauwi(a)-).

Zusammenfassend kann also gesagt werden, dass die zuletzt herangezogenen lp. Wörter - wie man sie auch sonst beurteilen mag - die hier aufgestellte germ. Etymologie für osfi. naava nicht beeinträchtigen können.

JoRma KoIvulehto

${ }^{1}$ Ich verweise hier auch auf $\operatorname{lpN}$ njap'pet 'jerk (at), twitch (at) / rykke i, nappe i' (Nielsen), das lautlich fi. nypätä (nyppiä)'raufen, zupfen, rupfen' entspricht (SKES); nypätä verhält sich nun zu germ. *hnuppö(ja)- (> ags. hnoppian 'pflücken, reissen)' wie fi. hypätä (hyppiä) 'springen, hüpfen', (dial.) 'tanzen' zu germ. *huppō(ja)- (> awn. hoppa 'hüpfen, tanzen'). Fi. nypätä kann zwar als deskriptiv gelten, aber auch deskriptive Wörter sind entlehnt worden (z.B. fi. napata 'erhaschen' aus schwed. nappa: SKES). Die genaue Übereinstimmung im Lautlichen und die Tatsache, dass die fi. und germ. Verben je denselben morphologischen Typus vertreten, lässt die Erklärung, es handle sich nur um Zufall, gesucht erscheinen (vgl. meinen Artikel in Virittăjă 1974 S. 121). 